\title{
Forward screening for seedling tolerance to Fe toxicity reveals a polymorphic mutation in ferric chelate reductase in rice
}

Siriphat Ruengphayak ${ }^{1,2}$, Vinitchan Ruanjaichon ${ }^{3}$, Chatree Saensuk', Supaporn Phromphan ${ }^{1}$, Somvong Tragoonrung ${ }^{5}$, Ratchanee Kongkachuichai ${ }^{6}$ and Apichart Vanavichit ${ }^{1,3,4^{*}}$

\begin{abstract}
Background: Rice contains the lowest grain Fe content among cereals. One biological limiting factor is the tolerance of rice to Fe toxicity. Reverse and forward genetic screenings were used to identify tolerance to Fe toxicity in $4,500 \mathrm{M}_{4}$ lines irradiated by fast neutrons (FN).

Findings: Fe-tolerant mutants were successfully isolated. In the forward screen, we selected five highly tolerant and four highly intolerant mutants based on the response of seedlings to 300 ppm Fe. Reverse screening based on the polymorphic coding sequence of seven Fe homeostatic genes detected by denaturing high performance liquid chromatography (dHPLC) revealed MuFRO1, a mutant for OsFRO1 (LOC_Os04g36720). The MuFRO1 mutant tolerated Fe toxicity in the vegetative stage and had $21-30 \%$ more grain Fe content than its wild type. All five highly Fe-tolerant mutants have the same haplotype as the MuFRO1, confirming the important role of OsFRO1 in Fe homeostasis in rice.

Conclusions: FN radiation generated extreme Fe-tolerant mutants capable of tolerating different levels of Fe toxicity in the lowland rice environment. Mutants from both reverse and forward screens suggested a role for OsFRO1 in seedling tolerance to Fe toxicity. The MuFRO1 mutant could facilitate rice production in the high-Fe soil found in Southeast Asia.
\end{abstract}

Keywords: Rice; Fe-tolerant mutants; Iron toxicity; OsFRO1; Fe homeostasis

\section{Findings}

Fe toxicity tolerance and grain Fe content

Fe toxicity is a serious agricultural problem, particularly when plants are grown in acidic soils (Quinet et al. 2012). More than 100 million hectares of lowland rice production on low-pH soil in Southeast Asia is limited by iron toxicity (Becker and Asch 2005). Fe toxicity can occur in flooded soils with a $\mathrm{pH}$ below 5.8 under aerobic conditions, and at a pH below 6.5 under anaerobic conditions (Fageria et al. 2008). Plants grown under such conditions accumulated two-fold more Fe in their leaves

\footnotetext{
* Correspondence: vanavichit@gmail.com

${ }^{1}$ Rice Science Center, Kasetsart University, Kamphaengsaen, Nakhon Pathom 73140, Thailand

${ }^{3}$ Rice Gene Discovery, National Center for Genetic Engineering and Biotechnology (BIOTEC), National Science and Technology Development Agency (NSTDA), Kasetsart University, Kamphaengsaen, Nakhon Pathom 73140, Thailand

Full list of author information is available at the end of the article
}

(Bashir et al. 2014). In low pH paddy field, anaerobic condition leads to the reduction of $\mathrm{Fe}^{3+}$ to $\mathrm{Fe}^{2+}$, resulting in excessive Fe availability and increased absorption (Quinet et al. 2012).

Genetic variation for tolerance to Fe toxicity exists in local landraces. However, most of their adaptive mechanism is associated with genetic variation in avoidance to Fe absorption and resulting in low grain Fe density. That association limits the chance for improving grain $\mathrm{Fe}$ density in acid soil, where high levels of $\mathrm{Fe}^{+2}$ are available for uptake and translocation to the grain. Therefore, it is important to understand natural genetic variation in enriching grain Fe density under Fe toxicity. One of the tolerance mechanisms that reduce excess $\mathrm{Fe}$ absorption is by reducing $\mathrm{Fe}^{+2}$ concentrations in rhizosphere by increasing the oxidative capability of roots (Ando 1983) or by excluding Fe from the rhizosphere (Tadano 1976). Another possible mechanism is

\section{空}

(C) 2015 Ruengphayak et al.; licensee Springer. This is an Open Access article distributed under the terms of the Creative Commons Attribution License (http://creativecommons.org/licenses/by/4.0), which permits unrestricted use, distribution, and reproduction in any medium, provided the original work is properly credited. 
increasing tissue tolerance to excessive levels of $\mathrm{Fe}^{+2}$ while increasing the rate of mobilization to grains. Such tolerance rice may link to Fe homeostasis that is not easily identified in existing germplasm. Therefore, one strategy is to find double mutation combining moderateto-high grain Fe content under neutral $\mathrm{pH}$ soil conditions while maintaining in the ability to withstand Fe-toxic conditions. These mutants may be likely to gain more $\mathrm{Fe}^{+2}$ to transport excessive Fe to grains.

\section{FN mutant library}

The fast neutron library was developed from Jao Hom Nin (JHN), a photoperiod non-sensitive, purple rice variety. By taking advantages of its distinctive color of leaves and grains, semi-dwarfism, early flowering and nutrient- rich grains, such mutant population is valuable for discovering mutation expressing useful genetic variation for both agronomic and nutritive characteristics. With its high combining ability, JHN mutants could be utilized as sources of new traits for marker-assisted selection in rice. Approximately 100,000 breeder seeds from JHN were mutagenized by using 33 Gy fast neutrons (FN). Successive generations from $M_{1}-M_{4}$, family history was traceable from individual $M_{1}$ plant. Due to abnormal mutation affecting seed set, several families were terminated leaving only 21,024 $\mathrm{M}_{4}$ mutant families forming the base population for genetic screening (Rice Science Center, Kasetsart University, Thailand). For Fe toxicity screening, 4,500 lines were randomly chosen for forward screening while 500 pooled DNA libraries (representing $24 \mathrm{M}_{1}$ plants per pool) from the $\mathrm{M}_{4}$ generation were used for reverse screening (Figure 1).

\section{Forward screening identified Fe toxicity tolerant mutants}

The 4,500 lines were screened in Fe-toxic $(\mathrm{pH} 3.0$, FeEDTA $300 \mathrm{ppm}$ ) nutrient solution at the five-leaf seedling stage. The low $\mathrm{pH}$ nutrient solution released excessive ferrous $\mathrm{Fe}^{+2}$, resulting in extensive leaf bronzing. The base population was assessed using a leaf bronzing index (LBI) (Arbeit 2003) in a time-course manner

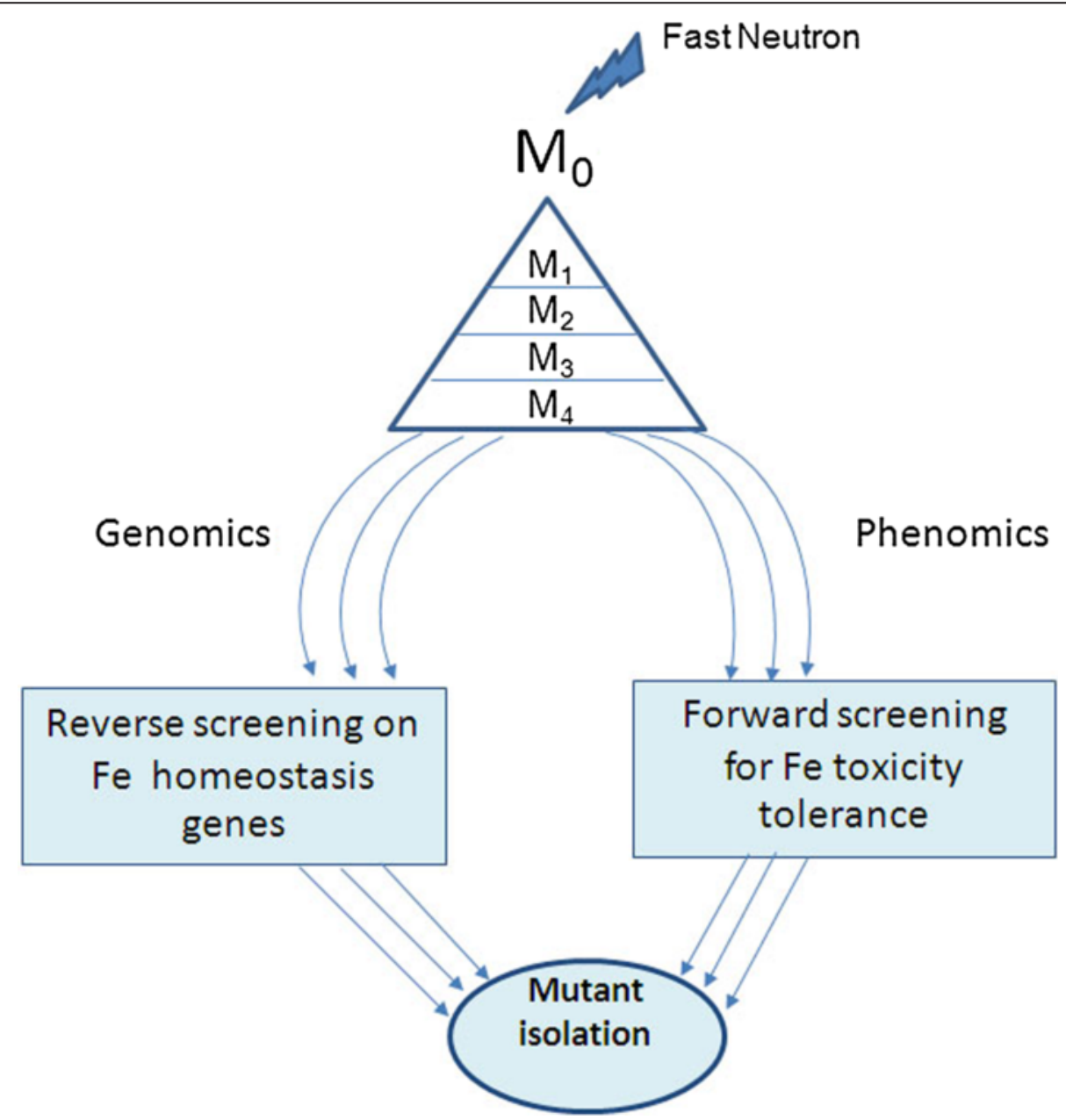

Figure 1 Schematic view of mutant discovery for rice mutant tolerance to Fe toxicity by reverse and forward genetic screens of a large FN-treated population. 

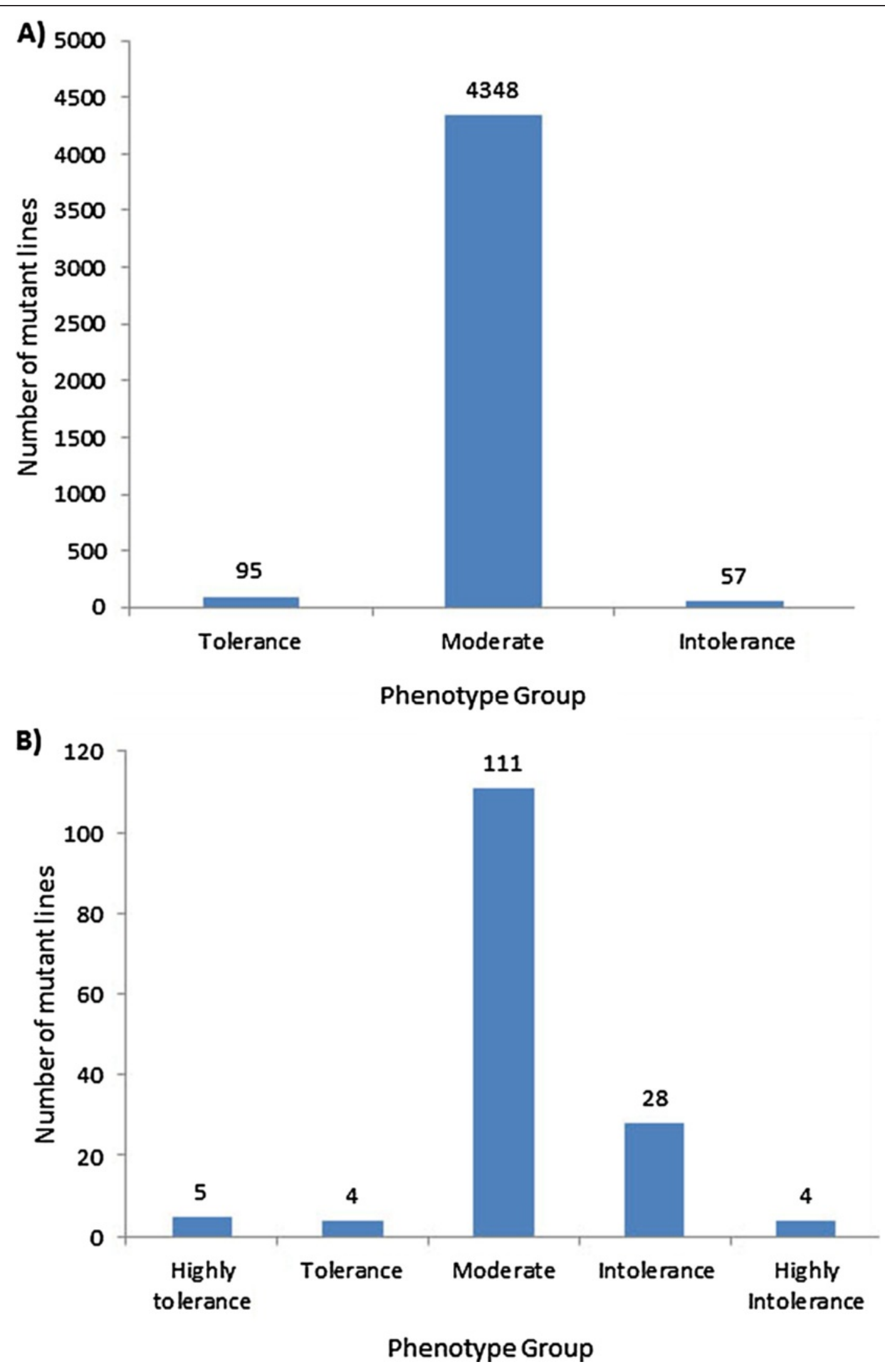

Figure 2 (See legend on next page.) 
(See figure on previous page.)

Figure 2 Distribution of responses to Fe toxicity of 4,500 $\mathrm{M}_{4}$ lines in Fe-toxic (pH 3.0, FeEDTA $\left.300 \mathrm{ppm}\right)$ nutrient solution at the five-leaf seedling stage. The outcome of $\mathbf{A}$ ) the 1 st round of screening and $\mathbf{B}$ ) the 2nd round of screening on $152 \mathrm{M}_{4}$ mutants consisting of 95 tolerant and 57 intolerant $\mathrm{M}_{4}$ lines selected from the first round of mutants.

following the death of the wild type JHN seedlings. In addition, the localization of iron in various parts of the leaf was visualized by PPB staining (Prom-U-thai et al. 2003). The days-to-seedling-death (DSD) of each variety was also recorded every other day. Phenotypic responses to Fe toxicity were categorized into tolerant, moderate and intolerant based on LBI and leaf PPB. Rice grains were also stained with PPB to reveal the embryonic Fe content, which is strongly associated with the grain $\mathrm{Fe}$ density. The first round screening of the $4,500 \mathrm{M}_{4}$ families yielded 95 tolerant and 57 intolerant mutants (Figure 2A). After repeated screenings, only 9 tolerant and 32 intolerant lines remained (Figure 2B). Selected mutants were scored for LBI every other day under the Fe toxic treatment (Figure 3). The result showed that the LBI of each mutant line increased rapidly for 20 days after Fe toxicity treatment, but the rate of increase can clearly be divided into tolerant and intolerant groups. By the 5th day, some intolerant mutant lines began to die, while the tolerant lines by the 11th day. The tolerant Mu783 prolonged seedling death to 19 days.

\section{Designing the reverse screen}

Mutant lines identified by forward screening can be used to develop functional markers for marker-assisted breeding. However, gene identification via forward mutant screen is complex, as FN-induced mutagenesis hits multiple targets. By reverse genetics, putative mutants carrying the candidate allele can be directly screened for the target phenotypic changes. This approach is called TILLING, Targeted Induced Local Lesion in Genome (Till et al. 2003). Using TILLING, seven candidate genes for iron homeostasis were selected, including ferric chelate reductase1 (OsFRO1; [MSU: LOC_Os04g36720]), ferritin 1 (OsFer1; [MSU: LOC_Os11g01530]), ferritin 2 (OsFer2; [MSU: LOC_Os12g01530]), iron regulated transporter 1 (OsIRT1; [MSU: LOC_Os03g46470]), nicotianamine synthase 3 (OsNAS3; [MSU: LOC_Os07g48980]), frataxin (OsFx; [MSU: LOC_Os01g57460]) and yellow stripe leaf 16 (OsYSL16; [MSU: LOC_Os04g45900]) (Gross et al. 2003 and Kawahara et al. 2013) for reverse screening using Denaturing High Performance Liquid Chromatography (DHPLC).

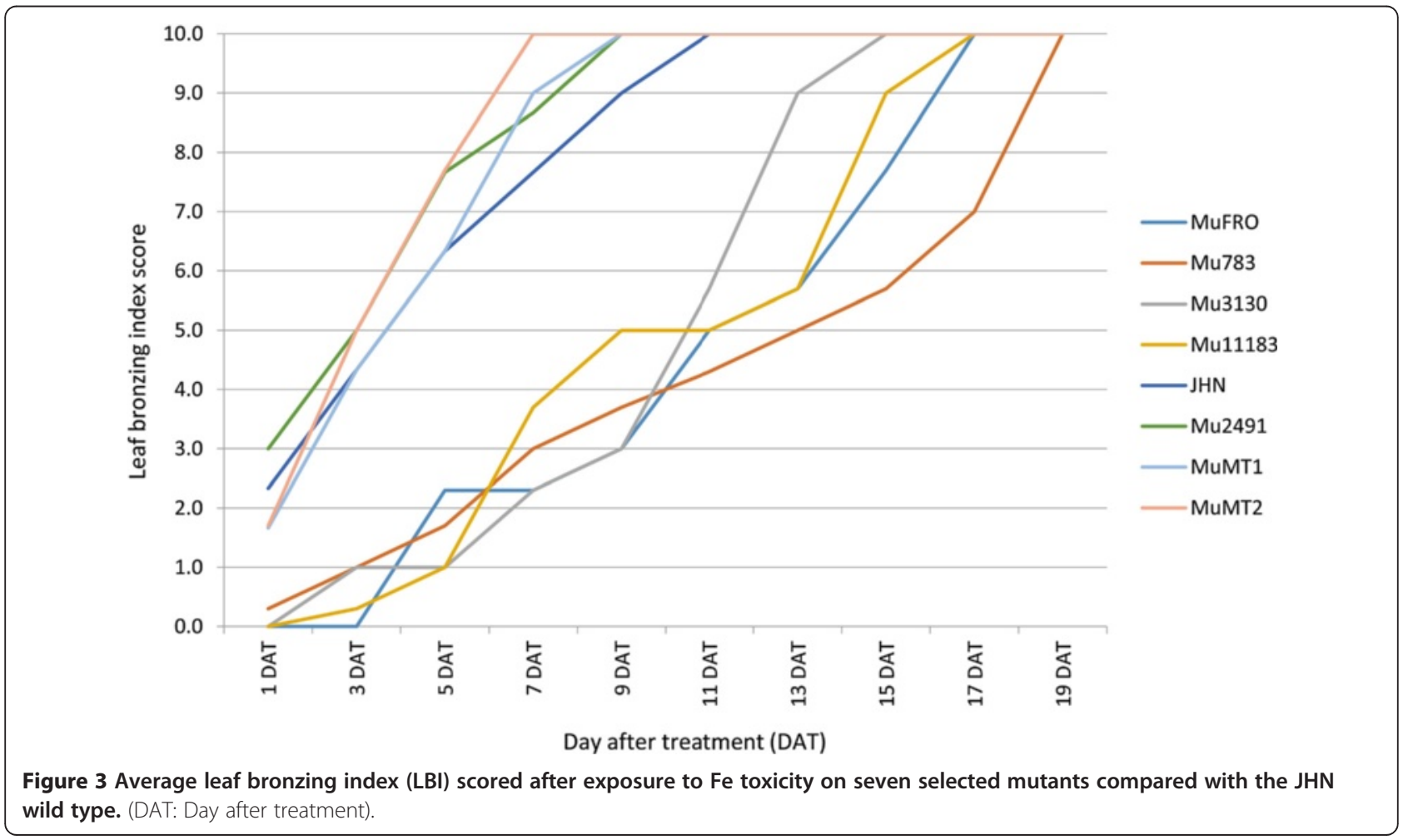




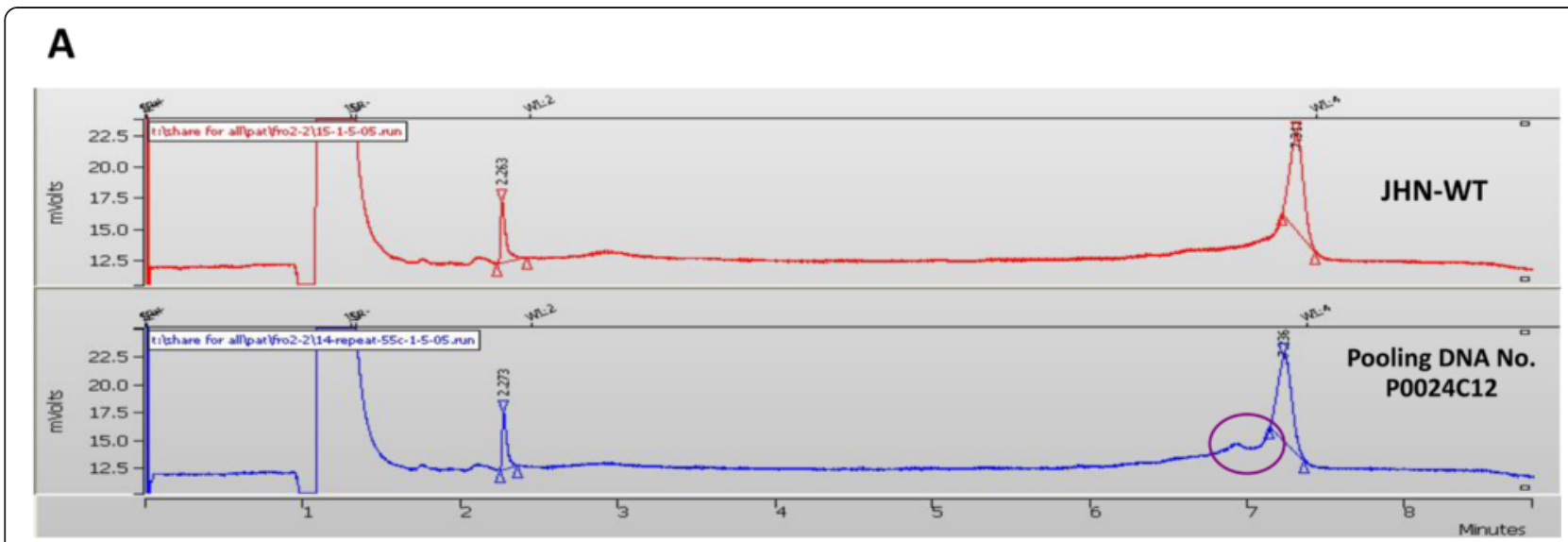

\section{B}

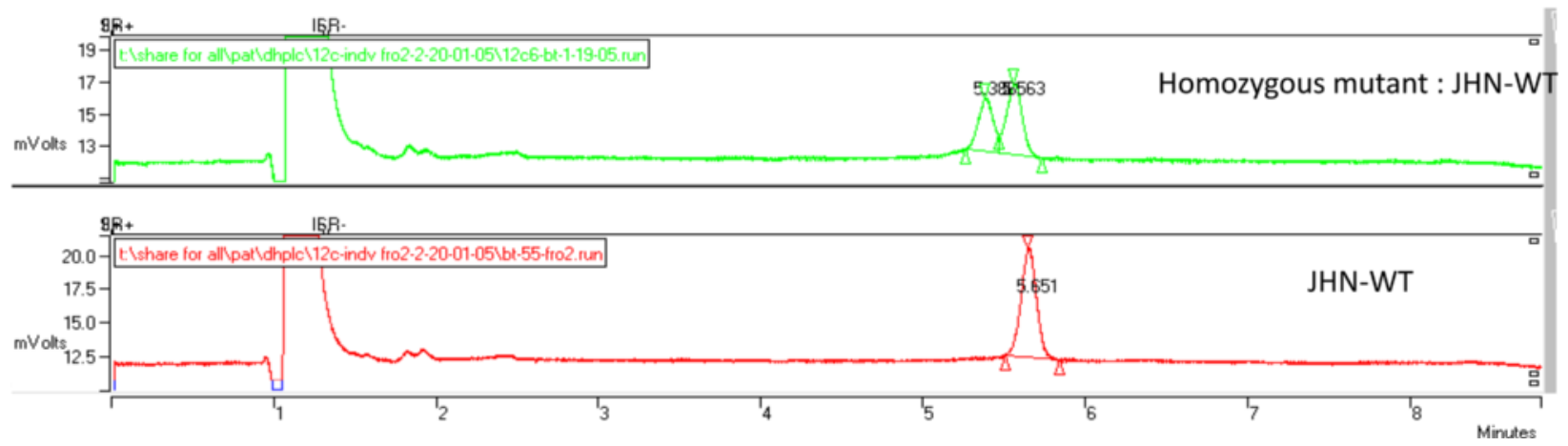

Figure 4 The dHPLC chromatograms of the OsFRO1 amplicons. A) The heteroduplex chromatogram was identified on 1D-DNA pooling No. P0024C12 (ratio1:24) compared to JHN-WT. B) The individual mutant line, a member of DNA pool No. P0024C12 that contained the mutant genotype, was identified in a 1:1 admixture with JHN WT.

Gene-specific primers were designed for polymorphic coding sequences of the seven candidate genes for reverse screening. Potential mutable sequence variations were identified for each candidate gene and queried for potential SNV via public domains. Selected differential genotypes for grain Fe density, including Xua Bue Nuo (XBN), JHN, IR68144, RB\#3, KDML105 (KD),
Azucena (Azu) and Nipponbare, were genotyped for the SNVs, which may be associated with tolerance to $\mathrm{Fe}$ toxicity (Additional file 1: Table S1). Primer pairs for PCR amplification and denaturing conditions of each mutable site for dHPLC are listed (Additional file 2: Table S2). Before injection into the dHPLC column, PCR amplicons were denatured at $95^{\circ} \mathrm{C}$ for $5 \mathrm{~min}$

Table 1 Haplotype variation of OsFRO1 in MuFRO1 compared to tolerant (KDML105) and intolerant (IR68144) controls

\begin{tabular}{|c|c|c|c|c|c|c|}
\hline \multirow{2}{*}{$\begin{array}{l}\text { Location on } \\
\text { gene structure }\end{array}$} & \multirow[t]{2}{*}{ Position on MSU7 } & \multicolumn{4}{|c|}{ Rice varieties } & \multirow{2}{*}{$\begin{array}{l}\text { OryzaSNP } \\
\text { @MSU ID }\end{array}$} \\
\hline & & $\mathrm{JHN}$ & MuFRO1 & IR68144 & KDML105 & \\
\hline Intron2 & Between 22183415\&22183416 & AAA & - & AAA & & - \\
\hline Intron2 & 22183525 & G & A & G & A & TBGI203991 \\
\hline Intron3 & 22183880 & A & G & A & G & - \\
\hline Intron3 & 22183900 & C & $\mathrm{T}$ & C & $\mathrm{T}$ & - \\
\hline Intron3 & 22184066 & c & $\mathrm{T}$ & c & T & - \\
\hline Exon4 (V > I) & 22184296 & G & A & G & A & - \\
\hline Exon5 ( $\mathrm{S}>\mathrm{C}$ ) & 22184982 & c & G & c & G & TBGI203998 \\
\hline
\end{tabular}


and annealed gradually from $95^{\circ} \mathrm{C}$ to $65^{\circ} \mathrm{C}$ over $30 \mathrm{~min}$ (Callery et al. 2006).

\section{Reverse screen by heteroduplex}

Reverse screen was initiated among $192 \mathrm{M}_{4}$ genomic DNA pools. Each pool represented either a set of $24 \mathrm{M}_{1}$ lines, or the total 4,608 lines. Amplified target fragments from the six candidate genes were screened for heteroduplexes using dHPLC in a 1:1 admixture with the control (wild type) amplicons. The results indicated that heteroduplex was only detected on OsFRO1 amplicons in DNA pool no.P024C12 (Figure 4A). Individual members of the $\mathrm{P} 024 \mathrm{C} 12$ pool were analyzed for potential mutants. Heteroduplex-forming amplicons were confirmed by sequencing (Figure 4B). No mutation was found in the remaining candidate genes. However, we cannot rule out the possibility of mutations within other parts of the candidate genes, such as introns and promoters that were not included in the design.

\section{Mutation on OsFRO1}

The OsFRO1 mutant was purified and designated 'MuFRO1'. The OsFRO1 target gene was confirmed by gene sequencing. Sequence comparison between wild type and MuFRO1 identified four new single nucleotide polymorphisms (SNPs) and one indel in several introns. Two single amino acid changes (SAP) were identified in Exons 4 and 5 (Table 1). One SAP on Exon 4 exhibited a Valine (V) to Isoleucine (I) change in the same hydrophobic group. Because the SAP is located within the ferric chelate reductase domain, the amino acid change may affect the functioning of OsFRO1 (Marchler-Bauer et al. 2013). OsFRO1 also contained an AAA deletion, a SNP in intron 2 and three SNPs in intron 3, similar to the mutations found in KDML105, the landrace Fe

Table 2 Bi-directional SNP primer sequences of OsFRO1

\begin{tabular}{ll}
\hline Primer name* & Sequence $\mathbf{5}^{\prime} \boldsymbol{\rightarrow} \mathbf{3}^{\prime}$ \\
\hline OsFRO1_Ex.4F & GGTGGATGAAGACACTACTGC \\
OsFRO1_Ex.4R & CACAGGACATTGGTCATAGCA \\
OsFRO1_Ex.4_SAP_A & GGCCTCCGGTTCGGATCGA \\
OsFRO1_Ex.4_SAP_G & GCCATGCAAAACAACCCGAC \\
OsFRO1_Ex.5F & TCATCTACTCTGTTTGGAGGT \\
OsFRO1_Ex.5R & CTTGCTGGCTTTGAGAAGACT \\
OsFRO1_Ex.5_SAP_G & TTCCTGAGGTTCTGGCAATG \\
OsFRO1_Ex.5_SAP_C & TGTCCACCTTGGCCCTGG \\
\hline
\end{tabular}

*Each SAP primer set was amplified using the KAPA 2 G Robust HS protocol (KAPABIOSYSTEMS, Woburn, USA) under the following thermal cycling conditions: one cycle at $95^{\circ} \mathrm{C}$ for $3 \mathrm{~min} ; 35$ cycles of $30 \mathrm{~s}$ denaturation at $95^{\circ} \mathrm{C}$, $30 \mathrm{~s}$ annealing at $61^{\circ} \mathrm{C}$, and $30 \mathrm{~s}$ extension at $72^{\circ} \mathrm{C}$; and a final extension at $72^{\circ} \mathrm{C}$ for 2 min. toxicity tolerant strain, but unlike JHN and IR68144, the intolerant varieties (Table 1). Therefore, there are multiple FN-induced SNVs in the mutant OsFRO1. We cannot rule out the possibility of finding more mutation on other part of the genome.

Ferric chelate reductase was first reported in Arabidopsis (Robinson et al. 1999) and later two FRO-like genes were identified in rice (Ishimaru et al. 2006). OsFRO1 was detected in leaves of $\mathrm{Zn}, \mathrm{Mn}$ and $\mathrm{Cu}$ deficient rice whereas OsFRO2 transcript was found on Fedeficient leaves but not in roots under Fe deficiency. The result indicated that rice posses a unique $\mathrm{Fe}^{2+}$ - uptake system via OsIRT1 and OsIRT2. A transgenic plant that fused refre1/372 from high $\mathrm{pH}$ tolerant yeast with the promoter of OSIRT1 showed strong increase in $\mathrm{Fe}^{3+}$ chelate-reductase activity and Fe-uptake rate than control under Fe-deficient conditions. When grown under calcareous soil with high $\mathrm{pH}$ and low Fe availability, the transgenic rice yielded 7.9 times more productive. Recently, subcellular localization of the FRO families from Arabidopsis were identified (Jain et al. 2014). AtFRO7, found in chloroplast, may play important roles in $\mathrm{Fe}$ transport into chloroplast whereas AtFRO3 and AtFRO8, found in mitochondria, may involve in mitochondrial metal ion homeostasis (Jain et al. 2014). Cellular function of OsFRO2 was recently identified as a membrane

Table 3 OsFRO1 haplotypes and grain PPB scores on Fe-tolerant mutants and standard rice cultivars

\begin{tabular}{|c|c|c|c|}
\hline \multirow[t]{2}{*}{ Variety } & \multirow{2}{*}{$\begin{array}{l}\text { OsFRO1 } \\
\text { Haplotype }\end{array}$} & \multicolumn{2}{|l|}{ Phenotype } \\
\hline & & PPB score & Fe toxicity \\
\hline MuFRO1 & $O S F R O 1^{A-G}$ & ++ & Highly tolerant \\
\hline Mu1463 & OSFRO1 ${ }^{\mathrm{A}-\mathrm{G}}$ & + & Highly tolerant \\
\hline Mu3130 & OsFRO1 $1^{\mathrm{A}-\mathrm{G}}$ & + & Highly tolerant \\
\hline Mu11183 & OSFRO1 $1^{\mathrm{A}-\mathrm{G}}$ & + & Highly tolerant \\
\hline Mu783 & OSFRO1 ${ }^{\mathrm{A}-\mathrm{G}}$ & + & Highly tolerant \\
\hline Mu2409 & OsFRO1 $1^{\mathrm{G}-\mathrm{C}}$ & + & Tolerant \\
\hline Mu2559 & OsFRO1 $1^{\mathrm{G}-\mathrm{C}}$ & + & Tolerant \\
\hline Mu2638 & OsFRO1 $1^{\mathrm{G}-\mathrm{C}}$ & + & Tolerant \\
\hline Mu3113 & OsFRO1 $1^{\mathrm{G}-\mathrm{C}}$ & + & Tolerant \\
\hline JHN & OsFRO1 $1^{G-C}$ & + & Moderate \\
\hline MuMT1 & OsFRO1 $1^{G-C}$ & ++ & Highly intolerant \\
\hline MuMT2 & OsFRO1 $1^{\mathrm{G}-\mathrm{C}}$ & ++ & Highly intolerant \\
\hline Mu2491 & OsFRO1 $1^{\mathrm{G}-\mathrm{C}}$ & + & Highly intolerant \\
\hline Mu3295 & OsFRO1 $1^{G-C}$ & + & Highly intolerant \\
\hline IR68144 & OsFRO1 $1^{\mathrm{G}-\mathrm{C}}$ & ++ & Highly intolerant \\
\hline KDML105 & OsFRO1 $1^{A-G}$ & 0 & Highly tolerant \\
\hline Pinkaset\#3 & OsFRO1 $1^{\mathrm{G}-\mathrm{C}}$ & 0 & Tolerant \\
\hline RIL 909-21-2-5 & OsFRO1 $1^{\mathrm{A}-\mathrm{G}}$ & 0 & Highly tolerant \\
\hline
\end{tabular}


Table 4 Single nucleotide variant (SNV) for type, the location and/or effect of each SNV on MuFRO1 compared with JHN

\begin{tabular}{|c|c|c|c|c|c|c|c|}
\hline LOC & Gene symbol & Chro. & Position on MSU7 & MuFRO1 & JHN-WT & SNP classification & Known SNVs \\
\hline LOC_Os02g02450 & OSYSLT & 2 & 863203 & C & $\mathrm{T}$ & Missense variant $(\mathrm{S}>\mathrm{L})$ & rs18774481 \\
\hline LOC_Os03g46470 & OsIRT1 & 3 & 26284263 & - & C & Intron & - \\
\hline LOC_Os04g36720 & OsFRO1 & 4 & Between $22183415 \& 22183416$ & - & AAA & Intron & - \\
\hline LOC_Os04g36720 & OsFRO1 & 4 & 22183525 & A & G & Intron & TBGI203991 \\
\hline LOC_Os04g36720 & OsFRO1 & 4 & 22183880 & G & A & Intron & - \\
\hline LOC_Os04g36720 & OsFRO1 & 4 & 22183900 & $\mathrm{~T}$ & C & Intron & - \\
\hline LOC_Os04g36720 & OsFRO1 & 4 & 22184066 & $\mathrm{~T}$ & C & Intron & - \\
\hline LOC_Os04g36720 & OsFRO1 & 4 & 22184296 & A & G & Missense variant & - \\
\hline LOC_Os04g36720 & OsFRO1 & 4 & 22184982 & G & C & Missense variant & TBGI203998 \\
\hline
\end{tabular}

All sequence variations were compared to reported SNVs in the Gramene (rs) and OryzaSNP (TBGI) databases.

bound protein in mitochondria (Emanuelsson et al. 2000). However, no report concerns the exact localization of OsFRO1 in rice and its role in Fe trafficking under Fe toxic conditions. Recently, transcriptomic analysis of rice grown under contrasting Fe levels revealed OsFRO2 was up-regulated under Fe deficiency but reverse under excessive $\mathrm{Fe}$ in shoots (Bashir et al. 2014). Furthermore, under Fe toxic condition, peroxidases, the enzyme known to cope with reactive oxygen species (ROS), was up-regulated in root (Quinet et al. 2012). Comparison between rice varieties with high and low grain Fe density, OsFRO1 expression in grains show no difference (Das et al. 2013). However, only OsFRO1 transcript was found in root of the high grain Fe density variety. This finding leads to more investigation on the role of OSFRO1 in enriching grain Fe content for rice grown under excessive Fe.

\section{Development of functional markers}

Marker-assisted selection is most efficient when the functional marker for a target trait is utilized. For Fe toxicity tolerance, the two non-synonymous SAPs on Exons 4 and 5 of the OsFRO1 gene are used as functional markers. To develop agarose-based, co-dominant markers for Fe toxicity tolerance, bi-directional PCR was developed (Table 2), combining four primers in a single PCR amplification (Liu et al. 1997). Target amplicons were detected by $1.2 \%$ agarose gel electrophoresis (Figure 5). These primer set and amplification protocols

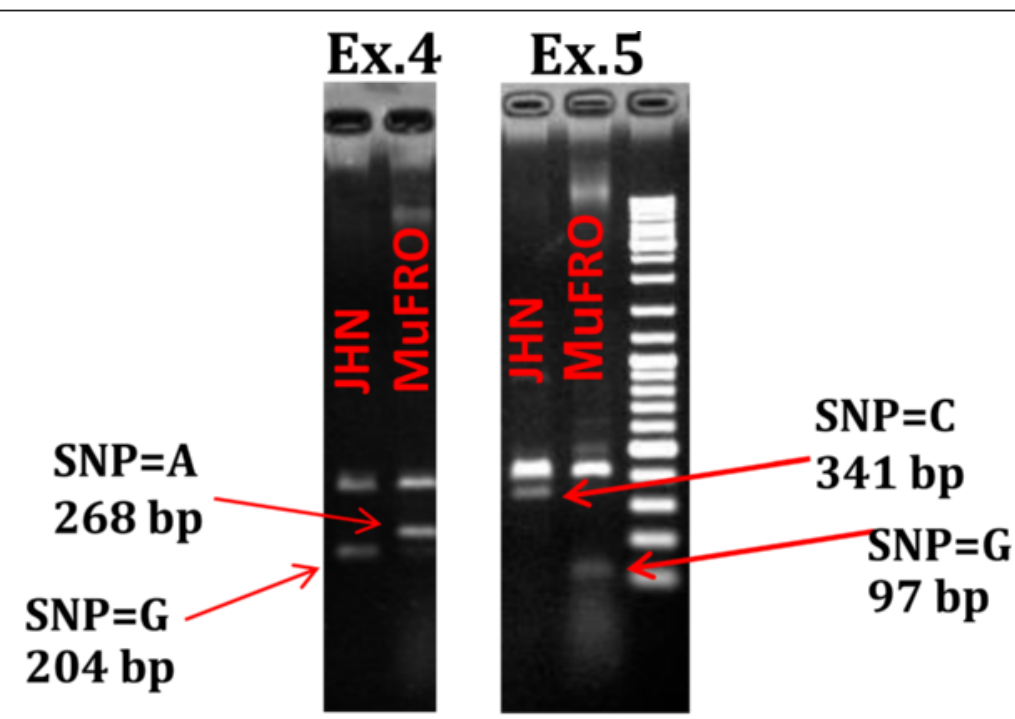

Figure 5 Genotyping of JHN and MuFRO1 using bi-directional SNP primers for a single amino acid polymorphism (SAP) in Exons 4 and 5. Expected amplicon size of the SAP in Exon 4: SAPEx.4 F/R = 435 bp, SAP_A/SAPEx. 4_R = 268 bp and SAPEx.4 F/SAP-G = 204 bp. Expected amplicon size of the SAP in Exon 5: SAPEx.5 F/R = 402 bp, SAPEx.5 F/SAP-C = 341 bp and SAP_G/SAPEx. 5_R=97 bp. 
Table 5 Fe contents (ppm) of JHN stem and leaf compared with MuFRO1 grown under control (4 ppm Fe) and Fe-toxic (300 ppm Fe) nutrient conditions

\begin{tabular}{llllllll}
\hline Variety & \multicolumn{1}{l}{ Control } & & & \multicolumn{2}{l}{ Toxic } & & \\
\cline { 2 - 3 } & Stem & Leaves & Total in shoot & & Stem & Leaves & Total in shoot \\
\hline JHN & $9.57 \pm 0.92$ & $17.72 \pm 0.64$ & $27.29 \pm 0.70$ & & $476.17 \pm 9.20$ & $371.68 \pm 9.21$ & $847.85 \pm 21.71$ \\
MuFRO1 & $35.52 \pm 1.15$ & $28.92 \pm 1.99$ & $64.44 \pm 2.59$ & & $204.18 \pm 6.11$ & $435.51 \pm 5.32$ & $639.69 \pm 10.76$ \\
\hline
\end{tabular}

can be used for marker-assisted selection to improve tolerance to Fe toxicity.

Two haplotypes of the two SAPs, 'A-G' and 'G-C', can differentiate the highly tolerant samples from the rest (Table 3). Genotyping of the 41 selected mutants for forward screening (Figure 2B) revealed four new highly Fe toxicity-tolerant mutants, Mu1463, Mu3130, Mu11183 and Mu783. Phenotypic screening confirmed mutants that were highly tolerant (5), moderate (1) and highly intolerant (4) to Fe toxicity. Such haplotypes can be directly applied to MAS for Fe toxicity tolerance.

In fast neutron treated population, it is not uncommon to identify multiple mutated genes from reverse screen. To ascertain if the multiple gene mutation was not false positive, we conducted a whole genome sequencing of the wild type JHN and extensive GBS of selected mutated genes using random lines core collection of the JHN mutant population to see if there have already existed in the JHN.

\section{Target sequencing on MuFRO1}

To further investigate the possibility of finding more candidate genes, targeted enrichment sequencing was conducted on 40 candidate genes that play roles in Fe transport from soil to seeds (Gross et al. 2003; Koike et al. 2004; Bashir et al. 2010; Masuda et al. 2012). The nucleotide sequence of 40 candidate genes $(240 \mathrm{~Kb})$ was collected for probe design (Additional file 3: Table S3). Targeted enrichment sequencing was conducted based on the Sure Select-XT Target enrichment system (Illumina paired end and multiplexed sequencing library by Agilent Technologies).

A new missense nucleotide variation was identified in OsYSL7, the metal-nicotinamide transporter protein (Table 4). The expression of OsYSL5, OsYSL6, OsYSL7, OsYSL14 and OsYSL17 were detected in epidermis, exodermis, cortex and stele of 3 week-old seedling root grown under Fe-deficient conditions for 2 weeks (Inoue et al. 2009). While, no expression was detected from maximum tillering to the flowering stages (Chandel et al. 2010).

\section{Phenotypic evaluation of MuFRO1}

Seeds of JHN and MuFRO1 harvested from two planting seasons were analyzed for $\mathrm{Fe}, \mathrm{Zn}$ and $\mathrm{Cu}$ contents using ICP-OES at the Institute of Nutrition, Mahidol University,

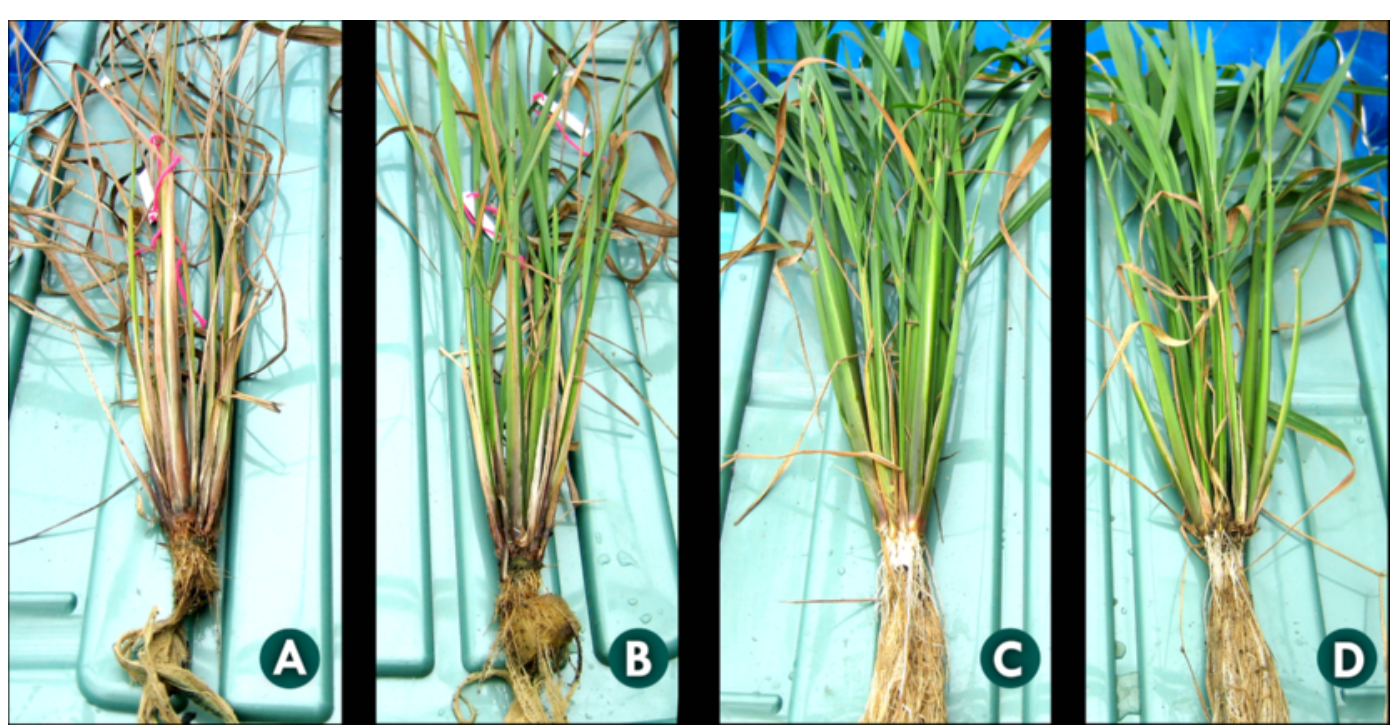

Figure 6 Rice plant treated with toxic nutrient solutions (300 ppm) for three weeks: A) wild type and B) MuFRO1 and under control (4 ppm) conditions: C) wild type and D) MuFRO1. 
Table 6 Iron and zinc contents in JHN and MuFRO1 brown rice seeds from two generations grown in normal soil conditions

\begin{tabular}{llll}
\hline No. & Variety & Fe $(\mathbf{p p m})$ & Zn $(\mathbf{p p m})$ \\
\hline 1 & MuFRO1 $\left(\mathrm{M}_{4}\right.$ seed $)$ & $13.7 \pm 0.38$ & $17.7 \pm 0.35$ \\
2 & $\mathrm{JHN}($ control $)$ & $10.5 \pm 0.44$ & $16.6 \pm 0.65$ \\
3 & MuFRO1 $\left(\mathrm{M}_{5}\right.$ seed $)$ & $11.7 \pm 0.30$ & $25.8 \pm 1.07$ \\
4 & JHN (control) & $9.4 \pm 0.25$ & $25.4 \pm 0.56$ \\
\hline
\end{tabular}

Thailand. The Fe toxicity hydroponic experiment was conducted to evaluate the effects of OsFRO1 on iron homeostasis. Five seedlings of MuFRO1 and JHN at the tillering stage were grown in normal $(\mathrm{pH} 5.5$, FeEDTA $4 \mathrm{ppm}$ ) and toxic (pH 3.0, FeEDTA 300 ppm) levels of Fe nutrient solution (Yoshida et al. 1976) for three weeks. The total Fe concentration in shoots was compared between MuFRO1 and JHN. The results indicated that under control conditions, MuFRO1 and JHN wild type contains $64.44 \pm 2.59 \mathrm{ppm}$ and $27.29 \pm 0.70 \mathrm{ppm}$ of total shoot Fe, respectively, or $136 \%$ higher than JHN whereas no substantial difference on their dry weights of the two samples. On the other hand, under Fe toxic conditions, the Fe concentrations in shoot of MuFRO1 and JHN were $639.69 \pm 10.76 \mathrm{ppm}$ and $847.85 \pm 21.71 \mathrm{ppm}$, respectively (Table 5). MuFRO1 remained green with more biomass (data not shown) than wild type (Figure 6). This result may suggest that MuFRO1 performed better Fe homeostasis by maintaining lower Fe content in the shoots. One such mechanism is simply by efficient partitioning into storage organelles like mitochondria and chloroplast. Therefore, OsFRO1 may play important roles in iron homeostasis and the maintenance of high biomass when grown under Fe toxicity conditions. JHN and MuFRO1 seeds were analyzed for $\mathrm{Fe}$ and $\mathrm{Zn}$ contents. MuFRO1 seeds contained $30 \%$ more grain iron than wild type JHN, but there was no difference in zinc content (Table 6). This opening a new opportunity to develop new rice varieties to withstand lowland Fe toxicity as well as enrichment of grain Fe density in the greater lowland rice growing area in the rice bowl of Asia.

\section{Additional files}

Additional file 1: Table S1. Natural sequence variation on two ferritin gene (OsFer1 and OsFer2) and Ferric chelate reductasel (OsFRO1) among selected varieties that differ in iron density.

Additional file 2: Table S2. Primer pairs and denaturing conditions for each mutable site.

Additional file 3: Table S3. Positions of 40 candidate genes (240 Kb) involving iron uptake, transport and storage in rice.

\section{Abbreviations}

AAS: Atomic Absorption Spectroscopy; DHPLC: Denaturing High Performance Liquid Chromatography; FN: Fast neutrons; ICP-OES: Inductively coupled optical emission spectrometry; JHN: Joa Hom Nin; LBI: Leaf bronzing index; MuFRO1: Ferric chelate reductase mutant; PPB: Perl Prussian Blue; SAP: Single amino acid polymorphism; SNP: Single nucleotide polymorphism; SNV: Single nucleotide variant; TILLING: Targeting Induced Local Lesions IN Genomes.

\section{Competing interests}

The authors declare that no competing interests exist.

\section{Authors' contributions}

SR and SP performed the Fe toxicity mutant screening experiments. RK analyzed the embryonic Fe density of selected mutant lines by ICP-OES. SR and VR characterized the OsFRO1 mutant and analyzed the data. ST

suggested the next-generation sequencing design. VR and CS performed target enrichment sequencing. The entire study was designed and coordinated by AV. SR drafted the manuscript and AV edited and improved the manuscript draft. All authors read and approved the final version of the manuscript.

\section{Acknowledgements}

This work was supported by the National Center for Genetic Engineering and Biotechnology (BIOTEC) and National Science and Technology Development Agency (NSTDA) Thailand (Grant No. P0010270), NSTDA Research Chair Grant (No. P12-01898) JST/BIOTEC (Grant No. P-12-01714) and the National Research Council of Thailand (NRCT) (Grant No.2547-113). SR gratefully acknowledges the financial support of the Royal Golden Jubilee (RGJ)-PhD program Grant No. PHD/0009/2546 from the Thailand Research Fund (TRF).

\section{Author details}

'Rice Science Center, Kasetsart University, Kamphaengsaen, Nakhon Pathom 73140, Thailand. ${ }^{2}$ Interdisciplinary Graduate Program in Genetic Engineering, Kasetsart University, Chatuchak, Bangkok 10900, Thailand. ${ }^{3}$ Rice Gene Discovery, National Center for Genetic Engineering and Biotechnology (BIOTEC), National Science and Technology Development Agency (NSTDA), Kasetsart University, Kamphaengsaen, Nakhon Pathom 73140, Thailand. ${ }^{4}$ Agronomy Department, Faculty of Agriculture at Kamphaengsaen, Kasetsart University, Kamphaengsaen, Nakhon Pathom 73140, Thailand. ${ }^{5}$ Genome Institute, National Center for Genetic Engineering and Biotechnology (BIOTEC), 113 Thailand Science Park, Phahonyothin Road, Khlong Nueng, Khlong Luang, Pathum Thani 12120, Thailand. Institute of Nutrition, Mahidol University, Phutthamonthon 4, Nakhon Pathom 73170, Thailand.

Received: 22 July 2014 Accepted: 11 December 2014

Published online: 20 January 2015

\section{References}

Ando T (1983) Nature of oxidizing power of rice roots. Plant Soil 72:57-71

Arbeit W (2003) Developing a standardized procedure to screen lowland rice (Oryza sativa) seedlings for tolerance to iron toxicity. MSc Thesis, Rheinischem Friedrich-Wilhelms University, Germany, p 46

Bashir K, Hanada K, Shimizu M, Seki M, Nakanishi H, Nishizawa NK (2014)

Transcriptomic analysis of rice in response to iron deficiency and excess. Rice 7:18

Bashir K, Ishimaru Y, Nishizawa NK (2010) Iron uptake and loading into rice grains. Rice 3:122-130

Becker M, Asch F (2005) Iron toxicity in rice-conditions and management concepts. J Plant Nutr Soil Sci 168:558-573

Callery PS, Boswell B, Gannett PM, Haining RL, Sanga M, Tirumalai P, Tracy TS (2006) dHPLC method for forensic DNA analysis. U.S. Department of Justice and prepared the following final report, $\mathrm{p} 17$

Chandel G, Banerjee S, Verulkar SB (2010) Expression profiling of metal homeostasis related candidate genes in rice (Oryza spp.) using semi quantitative RT-PCR analysis. Rice Genet Newsl 25:44-47

Das A, Sharma S, Mohapatra T (2013) An insight into differential Fe accumulation in developing rice grain through high throughput RNA-seq. 11th International Symposium on Rice Functional Genomics, November 20-23, New Delhi, India

Emanuelsson O, Nielsen H, Brunak S, von Heijne G (2000) Predicting subcellular localization of proteins based on their $\mathrm{N}$-terminal amino acid sequence. J Mol Biol 300(4):1005-1016 
Fageria NK, Santos AB, Barbosa Filho MP, Guimarães CM (2008) Iron toxicity in lowland rice. J Plant Nutr 31(9):1676-1697

Gross J, Stein RJ, Fett-Neto AG, Fett JP (2003) Iron homeostasis related genes in rice. Genet Mol Biol 26(4):477-497

Inoue H, Kobayashi T, Nozoye T, Takahashi M, Kakei Y, Suzuki K, Nakazono M, Nakanishi H, Mori S, Nishizawa NK (2009) Rice OsYSL15 is an iron-regulated iron (iii)-deoxymugineic acid transporter expressed in the roots and is essential for iron uptake in early growth of the seedlings. J Biol Chem 284:3470-3479

Ishimaru Y, Suzuki M, Tsukamoto T, Suzuki K, Nakazono M, Kobayashi T, Wada Y, Watanabe S, Matsuhashi S, Takahashi M, Nakanishi H, Mori S, Nishizawa NK (2006) Rice plants take up iron as an $\mathrm{Fe}^{3+}$-phytosiderophore and as $\mathrm{Fe}^{2+}$. Plant J 45:335-346

Jain A, Wilson GT, Connolly EL (2014) The diverse roles of FRO family metalloreductases in iron and copper homeostasis. Front Plant Sci 5(100):1-6 Kawahara Y, de la Bastide M, Hamilton JP, Kanamori H, McCombie WR, Ouyang S, Schwartz DC, Tanaka T, Wu J, Zhou S, Childs KL, Davidson RM, Lin H, Quesada-Ocampo L, Vaillancourt B, Sakai H, Lee SS, Kim J, Numa H, Itoh T, Buell CR, Matsumoto T (2013) Improvement of the Oryza sativa Nipponbare reference genome using next generation sequence and optical map data. Rice 6:4

Koike S, Inoue H, Mizuno D, Takahashi M, Nakanishi H, Mori S, Nishizawa NK (2004) OsYSL2 is a rice metal-nicotianamine transporter that is regulated by iron and expressed in the phloem. Plant J 39:415-424

Liu Q, Thorland EC, Heit JA, Sommer SS (1997) Overlapping PCR for bidirectional PCR amplification of specific alleles: a rapid one-tub method for simultaneously differentiating homozygotes and heterozygotes. Genome Res 7:389-398

Marchler-Bauer A, Zheng C, Chitsaz F, Derbyshire MK, Geer LY, Geer RC, Gonzales NR, Gwadz M, Hurwitz DI, Lanczycki CJ, Lu F, Lu S, Marchler GH, Song JS, Thanki N, Yamashita RA, Zhang D, Bryant SH (2013) CDD: conserved domains and protein three-dimensional structure. Nucleic Acids Res 41(D1):D348-D352

Masuda H, Ishimaru Y, Aung MS, Kobayashi T, Kakei Y, Takahashi M, Higuchi K Nakanishi H, Nishizawa NK (2012) Iron biofortification in rice by the introduction of multiple genes involved in iron nutrition. Sci Rep 2:543

Prom-u-thai C, Bernie D, Thomson G, Benjavan R (2003) Easy and rapid detection of iron in rice grain. ScienceAsia 29:203-207

Quinet M, Vromman D, Clippe A, Bertin P, Lequeux H, Dufey I, Lutts S, LefËVre I (2012) Combined transcriptomic and physiological approaches reveal strong differences between short- and long-term response of rice (Oryza sativa) to iron toxicity. Plant Cell Environ 35(10):1837-1859

Robinson NJ, Procter CM, Connolly EL, Guerinot ML (1999) A ferricchelate reductase for iron uptake from soils. Nature 397:694-697

Tadano T (1976) Studies on the methods to prevent iron toxicity in the lowland rice. Faculty Agric Hokkaido Univ 10(1):22-68

Till BJ, Reynolds SH, Greene EA, Codomo CA, Enns LC, Johnson JE, Burtner C, Odden AR, Young K, Taylor NE, Henikoff JG, Comai L, Henikoff S (2003) Large-scale discovery of induced point mutations with high-throughput TILLING. Genome Res 13:524-530

Yoshida S, Forno DA, Cock JH, Gomez KA (1976) Laboratory manual for physiological studies of rice, 3 rd edn. The International Rice Research Institute, Manila, Philippines, p 83

\section{Submit your manuscript to a SpringerOpen ${ }^{\circ}$ journal and benefit from:}

- Convenient online submission

- Rigorous peer review

- Immediate publication on acceptance

- Open access: articles freely available online

- High visibility within the field

- Retaining the copyright to your article

Submit your next manuscript at $>$ springeropen.com 